\title{
Ultrasonic Air Coupled Transducer Output Impedance Measurement Technique
}

\author{
Linas Svilainis $^{1}$, Andrius Chaziachmetovas ${ }^{1}$, Tomas E. Gomez Alvarez-Arenas ${ }^{2}$ \\ ${ }^{I}$ Department of Electronics Engineering, Kaunas University of Technology, \\ Studentu St. 50-340, LT-51368 Kaunas, Lithuania \\ ${ }^{2}$ Ultrasonic and Sensors Technologies Department, Spanish National Research Council (CSIC), \\ Serrano 144, 28006, Madrid, Spain \\ linas.svilainis@ktu.lt
}

\begin{abstract}
Estimation of the output impedance of ultrasonic transducers is required for electrical matching circuits design and for receiving amplifiers noise optimization. This paper presents a simple technique for transducer output impedance estimation using the same data acquisition system and signals that are conventionally used in non-destructive testing. A pair of ultrasonic transducers is used: one for transmission, one for reception. Received signal is recorded under low and high resistance load and transducer output impedance is then obtained from these two signals. Hence, this technique requires only one measurement channel and bias errors are low (no need for calibration). In addition, it is ground referenced and does not require the transducer to be removed from the positioning fixture. Experimental results obtained using different probing signals have been compared against conventional measurements obtained by an impedance analyser.
\end{abstract}

Index Terms-Amplifier; Output impedance measurement; Noise analysis; Noise density; Signal to noise ratio; Ultrasound transmission; Ultrasonic measurements.

\section{INTRODUCTION}

Ultrasonic techniques offer reliable tools for material integrity or properties evaluation in various fields [1]-[3]. Air-coupled ultrasound provides a further improvement since no liquid or dry coupling is required [4]-[7]. Unfortunately, mismatch of the acoustic impedance between most solid materials and air, together with propagation losses significantly reduces the amplitude of the received signal [8]. Signal losses are even higher, when it is transmitted through test material [9]. Therefore, a lot of research effort is concentrated on more efficient transducer design [10], [11] and high voltage excitation electronics [12], [13]. Previous research of the reception circuitry was concentrated on two issues: optimization of the input protection circuits [14]-[16] and noise reduction optimization [17], [18]. The case analysed here refers to thru-transmission spectroscopy [7], [9]; where protection circuits are not required. Furthermore, in order to reach best EMI performance and aiming for setup size reduction, preamplifier is placed immediately after transducer. Noise

Manuscript received 27 April, 2018; accepted 3 November, 2018.

This work was supported by ECERES grant (DPI2016-78876-RAEI/FEDER, UE) from the Spanish State Research Agency (AEI) and the European Regional Development Fund (ERDF / FEDER). analysis is based on theory presented by Motchenbacher in [19]. It includes the voltage and current noise sources of the preamplifier and transducer output impedance [20]. Then, in order to perform the required noise analysis, transducer output impedance is the only parameter that has to be measured. The transducers that motivate this work employ piezoelectric ceramics and composites. As the piezoelectric effect is assumed to be reciprocal transducer input impedance can be measured and then, assuming this reciprocity, it can be used as output impedance. Commercial impedance analysers are not always available; on a contrary, ultrasonic data acquisition systems [21]-[23] are more likely to be available in the case of ultrasonic non-destructive testing or imaging applications. Paper presents a technique for transducer output impedance measurement using conventional ultrasonic data acquisition systems. Novelty of the approach is that complex output impedance is obtained without the need of additional equipment. The technique was tested experimentally using different probing signals and impedance obtained was compared against the input impedance measured by the commercial impedance analyser.

\section{MeAsurement TeChNIQUE}

In general, ultrasonic imaging or measurement systems [21]-[23] structure can be analysed as presented in Fig. 1.

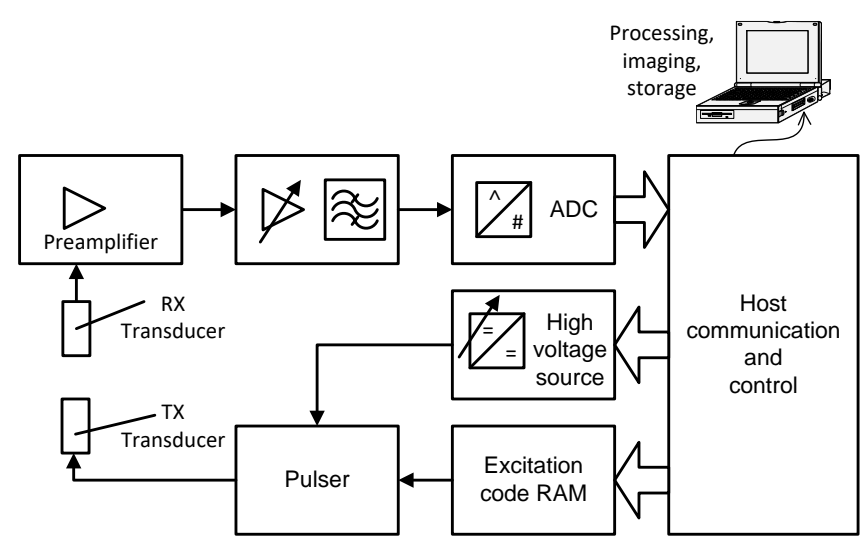

Fig. 1. Standard ultrasonic system structure.

The system contains an excitation channel, which is able to generate either single rectangular pulse or more complex rectangular waveform. If there is an excitation code memory, 
CW toneburst or chirp waveforms can also be generated. Received signals are amplified by low noise preamplifier and succeeding variable gain amplifier. The system contains an analog-to-digit converter (ADC) for signal digitization and further processing or storage. Ultrasonic transducers are used to convert the electrical energy into mechanical and vice versa [24]. Approaches like the microelectromechanical (MEMS), capacitive micromachined ultrasonic transducers (CMUTs) offer wide bandwidth, automated manufacturing and electronics integration [8]. Ferroelectret films are new active material [11]. Piezoelectric materials are most widely used for transducers. In most cases transducers are reciprocal devices and can be used both for transmitting pulses and receiving, where distinct transducers (thrutransmission or pitch-catch) or the same transducer (pulseecho) are used for transmission and reception.

Air-coupled transducers [10] that have been developed by Ultrasonic and Sensors Technologies Department, CSIC, were used in this research. These transducers are made using piezoelectric composite and a stack of resonant and nonresonant matching layers to match the transducer acoustic impedance to the air, canter frequency is $0.65 \mathrm{MHz}$ and bandwidth at $-20 \mathrm{~dB}$ in thru-transmission mode is $90 \%$.

The aim of the research was to evaluate the output impedance of these transducers.

\section{A. Impedance Measurement Techniques}

The input impedance can be estimated by subjecting the device under test to some excitation and then measuring the voltage on its clamps and the current passing through [25], [26]. Though there exist a large variety of impedance measurement techniques, I-V and auto-balancing bridge (ABB, Fig. 2) are the most popular thanks to their simplicity (I-V) or accuracy and wide range (ABB) [26].

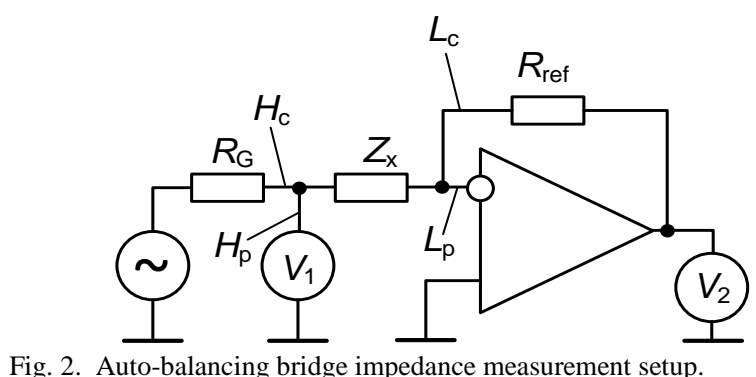

$\mathrm{ABB}$ uses virtual ground, therefore it is not suitable to measure the impedance of the transducer when it is fixed to scanners or alignment systems, because these elements are already grounded. In this case, transducer has to be removed from the system and detached from any ground connection for measurements. I-V technique uses current shunt reference resistance which is preferably connected to ground. High side current sensor can be used for I-V or RF $\mathrm{I}-\mathrm{V}$ measurement setup [27]. But both $\mathrm{I}-\mathrm{V}$ and $\mathrm{ABB}$ require two measurement channels which are usually not available on ultrasonic data acquisition system. Furthermore, the aforementioned techniques measure the input impedance, while we are interested in output impedance. As transducers were built using piezoelectric materials and the piezoelectric effect is supposed to be reciprocal, output impedance measurement using single acquisition channel would be preferred taking into account the ultrasonic acquisition system structure in Fig. 1.

\section{B. Proposed Technique}

The idea for the proposed technique is derived from Thevenin's analysis of the circuit presented in Fig. 3.

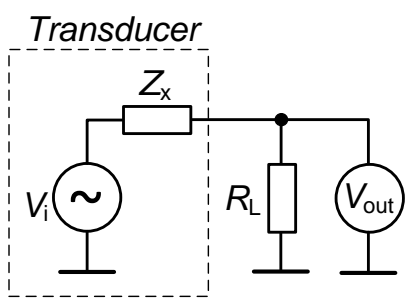

Fig. 3. Equivalent circuit of the transducer loaded by resistor.

There are few important conditions here: i) transducer must be driven by acoustic energy, generated by transmitting transducer (refer to Fig. 1); ii) distance and propagating conditions are not changing during the experiment; iii) load resistor $R_{L}$ is much lower that the input impedance of the transducer output voltage measurement circuit.

If two measurements are done using different $R_{L}$ values, $R_{L}$ (low resistance) and $R_{H}$ (higher resistance), then the voltage on the load can be expressed as:

$$
\begin{gathered}
V_{L}=k \frac{V_{i} R_{L}}{Z_{x}+R_{L}}, \\
V_{H}=k \frac{V_{i} R_{H}}{Z_{x}+R_{H}},
\end{gathered}
$$

where $V_{i}$ is the internal EMF, $k$ is the voltage measurement circuit transmission coefficient. Solving these equations for $Z_{x}$ and $V_{i}$ one can arrive to:

$$
\begin{gathered}
Z_{x}=\frac{\left(V_{H}-V_{L}\right) R_{L} R_{H}}{R_{H} V_{L}-R_{L} V_{H}}, \\
V_{i}=\frac{\left(R_{H}-R_{L}\right) V_{H} V_{L}}{k\left(R_{H} V_{L}-R_{L} V_{H}\right)} .
\end{gathered}
$$

It must be noted, that the transmission coefficient of the voltage measurement circuit vanished from (3). This means that the errors of the voltage measurement channel will not affect the result of $Z_{x}$ estimation.

\section{Output Impedance Measurement System}

A conventional ultrasonic data acquisition system [21] was used for the measurements. The system (Fig. 4) contains a rectangular high voltage signals generation pulser [13]. Both unipolar and bipolar single pulses of variable duration or sets of pulses with arbitrary width and spacing can be generated. Pulse amplitude can be programmed using a variable high voltage power source. Reception part contains a $100 \mathrm{Ms} / \mathrm{s} 10 \mathrm{bits}$ analog-to-digit converter (ADC) with $32 \mathrm{k}$ samples buffer memory. Control of the system is done by host PC via a high speed USB2 interface. The only modification used was the high input impedance, $40 \mathrm{~dB}$ gain preamplifier with connector for easy $R_{L}, R_{H}$ replacement. Four probing signal types were used: i) single rectangular pulse; ii) quasichirp (rectangular version of linear frequency 
modulation signal); iii) set of rectangular $\mathrm{CW}$ tonebursts with variable fill-in frequency and iv) $\mathrm{CW}$ sinusoid of variable frequency.

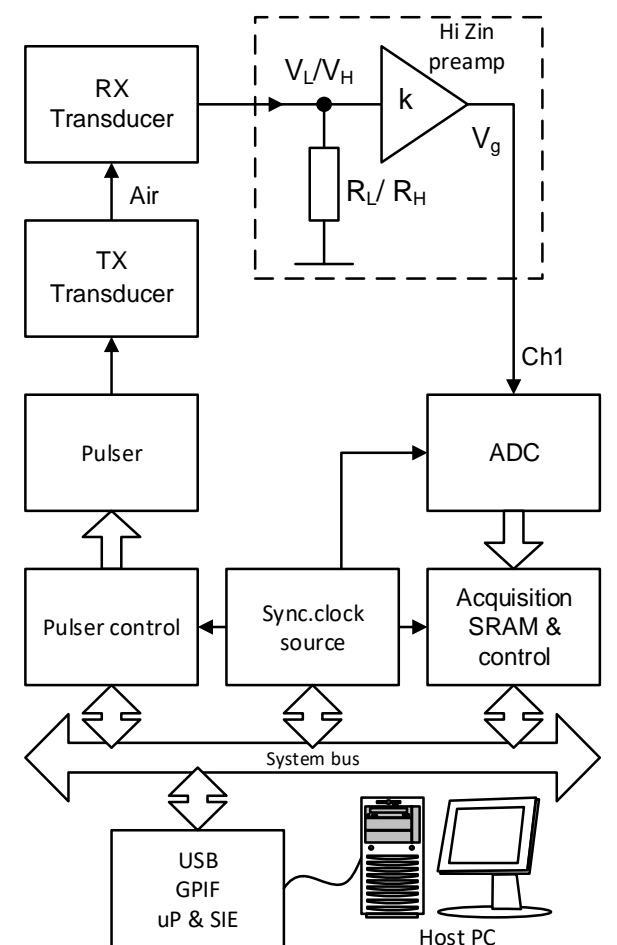

Fig. 4. Structure of the proposed output impedance measurement system.

Single pulse was chosen because it is most popular in ultrasonic NDT systems. Quasichirp is a spread spectrum signal type that is becoming popular both in ultrasonic imaging [27] and impedance measurement. It offers both wide bandwidth and good SNR in measurements at the expense of relatively simple excitation electronics (no linear amplifiers is required). However, duration (so the energy) of the chirp is limited by the propagation time of the second reflection traveling between the transducers. Duration of the CW toneburst was also limited by reflection traveling between the transducers, but it can deliver higher SNR thanks to concentration on a single frequency. CW sinusoid can deliver highest SNR: duration was deliberately not limited in order to evaluate whether overlap of the multiple reflections will influence the measurement. While the first three signals can be generated using conventional ultrasonic system, generation of $\mathrm{CW}$ sinusoid required a direct digital synthesizer and linear amplifier.

Response to pulse and chirp signals obtained at the ADC was converted to frequency domain using discrete Fourier transform (DFT). It was used as $V_{L}$ (when $R_{L}$ was used to load the transducer output) or $V_{L H}$ (in case of $R_{H}$ as a load), in (1), (2) for output impedance calculation. No scaling of DFT output was required, impedance AC response was obtained immediately. Toneburst and $\mathrm{CW}$ sinusoid signals required fundamental frequency amplitude extraction. Sine wave correlation (SWC) technique [20] was used to extract the complex voltage $V\left(V_{L}\right.$ or $V_{H}$ in (1), (2) accordingly)

$$
V=V_{c}-j V_{s}
$$

Sine and cosine functions were used to correlate the signal $y_{m}$, sampled at time instances $t_{m}$

$$
\left\{\begin{array}{l}
V_{c}=\frac{\sum_{m=1}^{M}\left[\cos \left(2 \pi f t_{m}\right) \times y_{m}\right]}{\sum_{m=1}^{M}\left[\cos \left(2 \pi f t_{m}\right)\right]^{2}}, \\
V_{s}=\frac{\sum_{m=1}^{M}\left[\sin \left(2 \pi f t_{m}\right) \times y_{m}\right]}{\sum_{m=1}^{M}\left[\sin \left(2 \pi f t_{m}\right)\right]^{2}},
\end{array}\right.
$$

where $f$ is the probing frequency, $m$ is the sample number and $M$ is the total number of samples used.

For ultrasonic data acquisition it is essential that both excitation sequence generator and reception ADC share the same synchronization clock. This property is important for SWC: then estimation of the probing frequency is not used.

\section{Sensitivity Analysis to Select Reference Loads}

Sensitivity analysis was carried out in order to evaluate the expected uncertainty of the measurements and to select the optimal load resistors. Sensitivity for voltage and load resistance can be found taking partial derivatives of (3):

$$
\begin{aligned}
& a_{V H}=\frac{\partial Z_{\text {out }}}{\partial V_{H}}=\frac{R_{L} R_{H}}{R_{H} V_{L}-R_{L} V_{H}}+\frac{R_{L}^{2} R_{H}\left(V_{H}-V_{L}\right)}{\left(R_{H} V_{L}-R_{L} V_{H}\right)^{2}}, \\
& a_{R H}=\frac{\partial Z_{\text {out }}}{\partial R_{H}}=\frac{R_{L}\left(V_{H}-V_{L}\right)}{R_{H} V_{L}-R_{L} V_{H}}-\frac{R_{L} R_{H}\left(V_{H}-V_{L}\right) V_{L}}{\left(R_{H} V_{L}-R_{L} V_{H}\right)^{2}}, \\
& a_{V L}=\frac{\partial Z_{\text {out }}}{\partial V_{L}}=\frac{R_{L} R_{H}}{R_{H} V_{L}-R_{L} V_{H}}-\frac{R_{L} R_{H}^{2}\left(V_{H}-V_{L}\right)}{\left(R_{H} V_{L}-R_{L} V_{H}\right)^{2}}, \\
& a_{R L}=\frac{\partial Z_{\text {out }}}{\partial R_{H}}=\frac{R_{H}\left(V_{H}-V_{L}\right)}{R_{H} V_{L}-R_{L} V_{H}}-\frac{R_{L} R_{H}\left(V_{H}-V_{L}\right) V_{L}}{\left(R_{H} V_{L}-R_{L} V_{H}\right)^{2}}
\end{aligned}
$$

Since the same measurement channel is used, then voltage measurement should be free from bias errors, therefore random errors should be summed by their power. Load resistance was not measured relying on specifications (1\%). The expanded uncertainty of the measurement then is

$$
\begin{aligned}
u_{e}= & 3 \sqrt{\left(a_{V H} \sigma_{V H}\right)^{2}+\left(a_{V L} \sigma_{V L}\right)^{2}}+ \\
& +\left|a_{R L} \varepsilon_{R L}\right|+\left|a_{R H} \varepsilon_{R H}\right| .
\end{aligned}
$$

Voltage measurement standard deviation can be estimated taking the integral over measurement bandwidth. In case of SWC, measurement bandwidth $d f$ is defined by sampling frequency $f_{s}$ and SWC samples number $M$

$$
d f=\frac{f_{s}}{M}
$$

$M$ was 5000 for $\mathrm{CW}$ toneburst measurements and 32000 for $\mathrm{CW}$ sinewave. This results in very narrow bandwidth, therefore measurement noise $\sigma_{V H}$ and $\sigma_{V L}$ can be obtained 
from total noise voltage density:

$$
\left\{\begin{array}{l}
\sigma_{V H}=d f \times e_{n t o t} @ R H \\
\sigma_{V L}=d f \times e_{n t o t} @ R L
\end{array}\right.
$$

Six components were considered: thermal noise $\left(e_{n T R}\right)$ of the transducer and load resistance $\left(e_{n R L}\right.$ or $\left.e_{n R H}\right)$, amplifier voltage noise $e_{n}$, voltage generated by current noise $i_{\mathrm{n}}$ on positive and negative input $\left(e_{n c R L-}\right.$ or $e_{n c R H-}$ and $\left.e_{n c-}\right)$ and quantization noise $\left(e_{n Q}\right)$ :

$$
\begin{gathered}
e_{n t o t @ R L}=e_{n T R}+e_{n R L}+e_{n}+e_{n c R L+}+e_{n c-}+e_{n Q}, \\
e_{n t o t @ R H}=e_{n T R}+e_{n R H}+e_{n}+e_{n c R H+}+e_{n c-}+e_{n Q} .
\end{gathered}
$$

More details on amplifier, used in experiments, noise evaluation can be found in [20]. Operational amplifier LMH6624 was used as preamplifier. It has $e_{n}=$ $0.92 \mathrm{nV} / \sqrt{\mathrm{Hz}}, \quad i_{n}=2.3 \mathrm{pA} / \sqrt{\mathrm{Hz}}$ noise densities. Feedback circuit resistance was $10 \Omega$. The quantization noise can be accounted by using ADC bits and reference voltage $V_{\text {ref }}$

$$
e_{n Q}^{2}=\frac{V_{r e f}^{2}}{0.5 f_{s} \times 2^{2 \times b i t s} 12} .
$$

Initial estimation of the transducer output impedance has been carried out by exciting it with $650 \mathrm{kHz}$ toneburst and measuring the output voltage in unloaded and loaded condition. Load resistance was varied until half of the open circuit voltage was achieved. The estimated impedance magnitude was $300 \Omega$. Simulation in Matlab of measurement using (1)-(16) was carried out in order to establish how do errors behave for different real and imaginary part combinations resulting to the same impedance magnitude $300 \Omega$. Random errors for the case $R_{L}=50 \Omega$ and $R_{H}=$ $330 \Omega$ are presented in Fig. 5 .

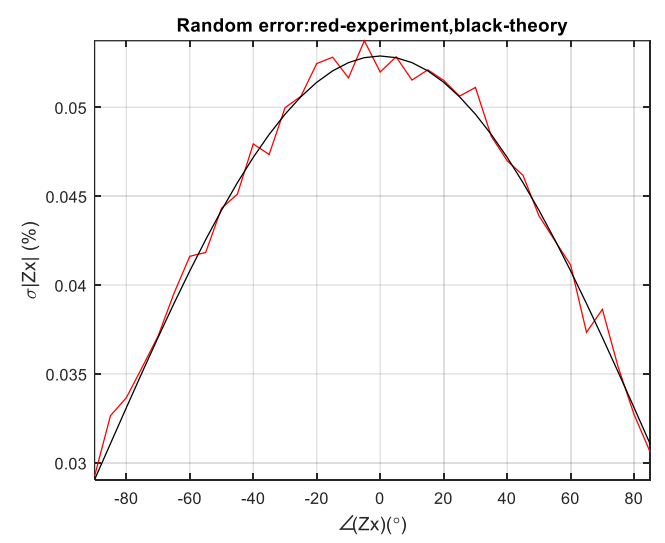

Fig. 5. $Z_{x}$ magnitude $(300 \Omega)$ estimation random errors vs. angle: red experiment, black - theory.

It can be seen that random errors vary almost twice. Impedance measured is complex. If impedance angle is

$$
\angle_{\text {Zout }}=\arctan \left(Z_{\text {out }}\right) \text {. }
$$

Then, sensitivity coefficients for the angle estimation are obtained by partial derivatives:

$$
\begin{aligned}
& a_{V H \angle}=\frac{\partial \angle_{\text {Zout }}}{\partial V_{H}}=\frac{a_{V H}}{\sqrt{\left(1+Z_{\text {out }}^{2}\right)}}, \\
& a_{R H \angle}=\frac{\partial \angle_{\text {Zout }}}{\partial R_{H}}=\frac{a_{R H}}{\sqrt{\left(1+Z_{\text {out }}^{2}\right)}}, \\
& a_{V L \angle}=\frac{\partial \angle_{\text {Zout }}}{\partial V_{L}}=\frac{a_{V L}}{\sqrt{\left(1+Z_{\text {out }}^{2}\right)}}, \\
& a_{R L \angle}=\frac{\partial \angle_{\text {Zout }}}{\partial R_{L}}=\frac{a_{R L}}{\sqrt{\left(1+Z_{\text {out }}^{2}\right)}} .
\end{aligned}
$$

Expanded uncertainty for impedance angle estimation is

$$
\begin{aligned}
u_{e}= & 3 \sqrt{\left(a_{V H \angle} \sigma_{V H}\right)^{2}+\left(a_{V L \angle} \sigma_{V L \angle}\right)^{2}}+ \\
& +\left|a_{R L \angle} \varepsilon_{R L \angle}\right|+\left|a_{R H \angle} \varepsilon_{R H}\right| \cdot .
\end{aligned}
$$

Simulation results for the angle errors are in Fig. 6.

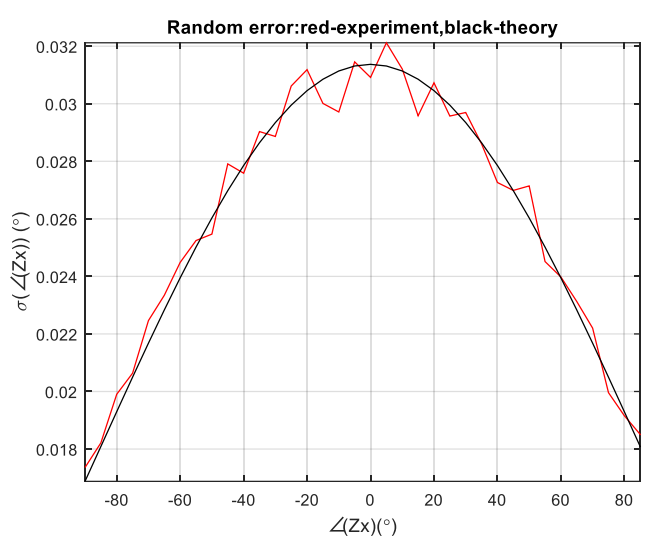

Fig. 6. $Z_{x}$ angle estimation random errors vs. angle: red - experiment black - theory.

Worst-case analysis for resistors errors was carried out by simulation of equations (1)-(22) in Matlab. It was found that bias errors are the largest when resistance deviation is of opposite sign for $R_{L}$ and $R_{H}$. Therefore, bias errors for resistance tolerance influence were replaced by simulation results. Simulation results of bias error for the case $R_{L}=$ $50 \Omega, R_{H}=330 \Omega$ presented in Fig. 7 (magnitude) and Fig. 8 (angle).

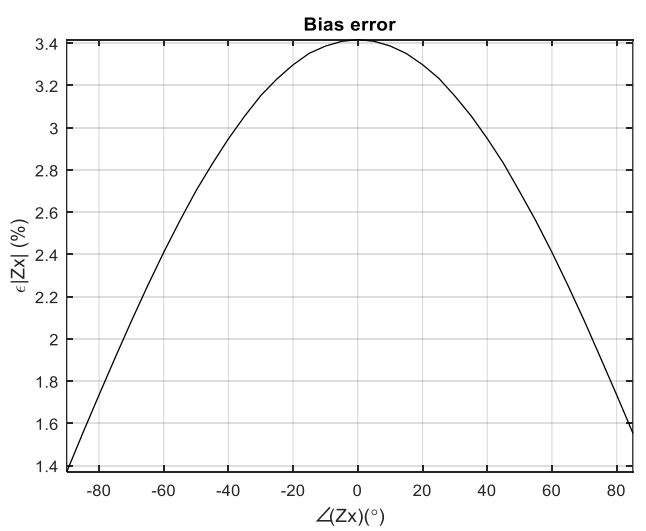

Fig. 7. $Z_{x}$ magnitude $(300 \Omega)$ estimation bias error vs. angle. 


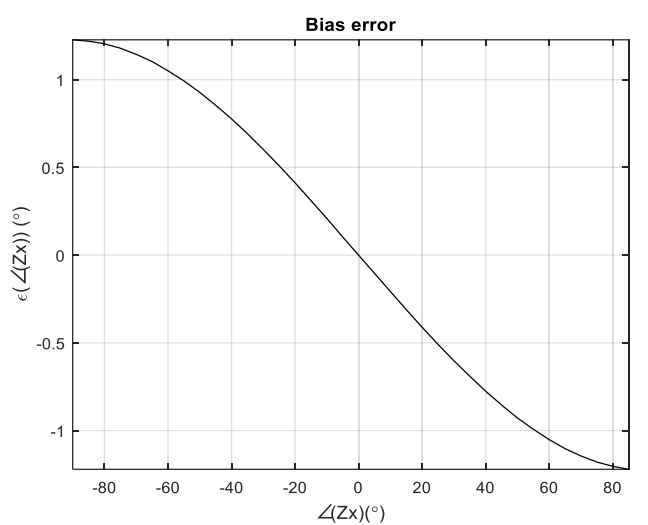

Fig. 8. $Z_{x}$ angle estimation bias angle error vs. angle.

Same simulation was used to analyse the errors obtained with different $R_{L}$ and $R_{H}$ values. $R_{L}$ was varied from $10 \Omega$ (to reduce contact resistance influence) to $\left|Z_{x}\right|(300 \Omega)$ and $R_{H}$ was varied from $\left|Z_{x}\right|(300 \Omega)$ to $100 \mathrm{k} \Omega$.

It can be seen that impedance estimation bias errors (magnitude: Fig. 9, angle: Fig. 10) are lower if $R_{H}$ value is significantly larger than $\left|Z_{x}\right|(20 \mathrm{k} \Omega$ and more in the case analysed).

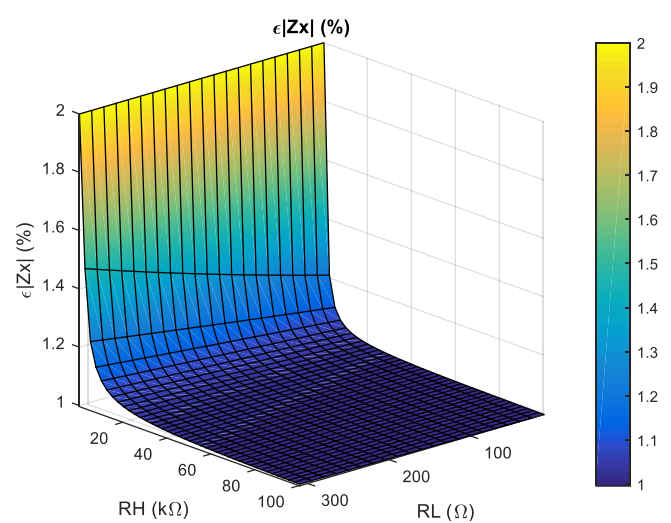

Fig. 9. $Z_{x}$ magnitude (300 $\Omega$ ) estimation bias error vs. $R_{L}$ and $R_{H}$ load.

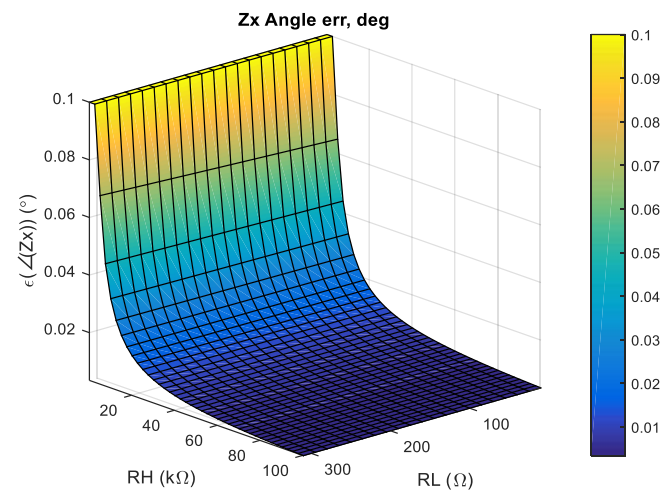

Fig. 10. $Z_{x}(300 \Omega)$ angle estimation bias error vs. $R_{L}$ and $R_{H}$ load.

It can be concluded, that large $R_{H}$ values reduce the bias error caused by $R_{H}$ deviation $(1 \%)$ and that the only influence of $R_{L}(1 \%)$ that still remains contributes to a $1 \%$ of the total error bias.

Meanwhile, random error of the impedance magnitude (Fig. 11) and the angle (Fig. 12) estimation were very much affected by low $R_{H}$ values.

This can be explained by the signal level reduction at low $R_{L}$ values (down to $-30 \mathrm{~dB}$ ) which is not compensated by noise reduction (only $-3 \mathrm{~dB}$ ). Optimum $R_{L}$ value was equal to $e_{n} / i_{n}$ as can be predicted by theory [19]. Concurrently, $R_{H}$ value should be as high as possible in order to maximize the output amplitude. It should be noted that estimation errors are not very sensitive to $R_{L}$ and $R_{H}$ choice: reduction is from $2 \%$ to $1 \%$ for bias errors and from $0.02 \%$ to $0.014 \%$ for random errors.

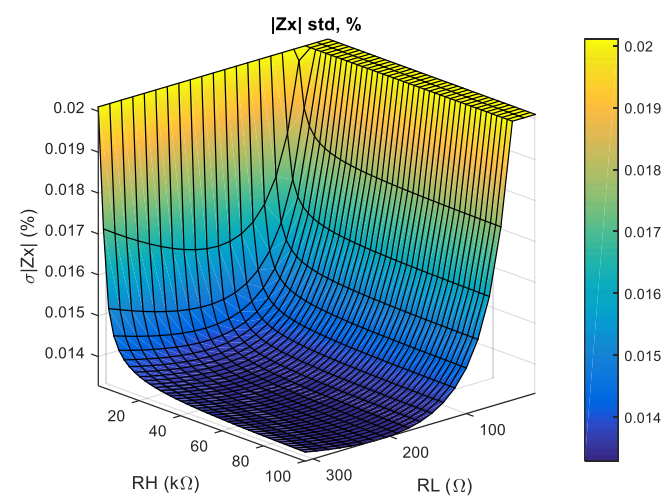

Fig. 11. $Z_{x}$ magnitude (300 $\Omega$ ) estimation random error vs. $R_{L}$ and $R_{H}$ load

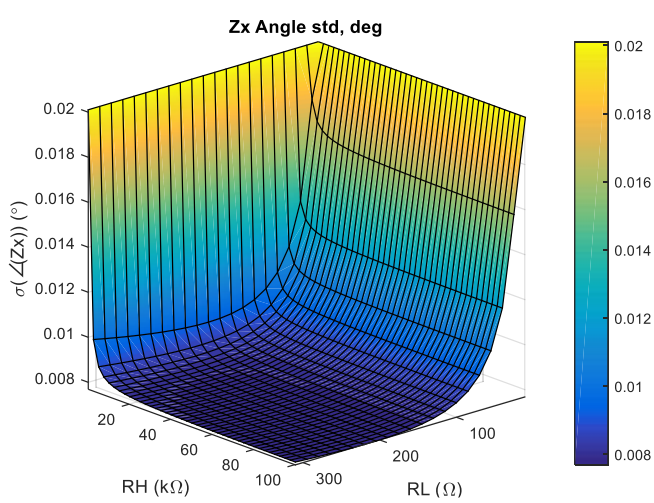

Fig. 12. $Z_{x}(300 \Omega)$ angle estimation random error vs. $R_{L}$ and $R_{H}$ load.

It should be further noticed that analysed error sources provide acceptable accuracy (expanded uncertainty $3 \%$ ) at analysed point $\left(\left|Z_{x}\right| 300 \Omega\right)$. Uncertainty within $5 \%-10 \%$ is acceptable for transducer evaluation as the impedance of the transducer [24] varies with temperature, and the transmission over the air is also affected by other factors like the air flow.

\section{EXPERIMENTAL RESULTS}

Two aforementioned air coupled transducers were placed against each other at $20 \mathrm{~mm}$ distance (Fig. 13).

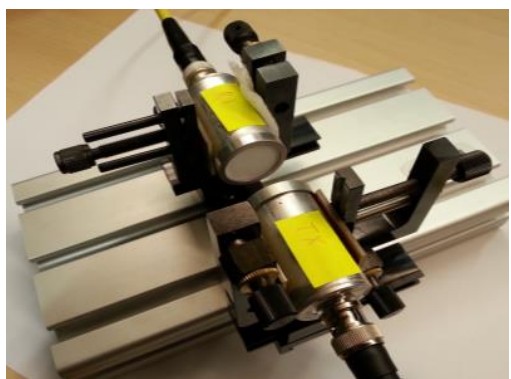

Fig. 13. Transducers' arrangement used in experiments.

Each transducer was attached to a goniometric test fixture and they were aligned for maximum level of second reflection. Several load resistors $R_{L}$ and $R_{H}$ were used: $50 \Omega$, $330 \Omega, 10 \mathrm{k} \Omega$, and $100 \mathrm{k} \Omega$. Transducer input impedance was also measured using the 6530B series (Wayne Kerr Electronics, UK) precision impedance analyser (specified 
uncertainty $\pm 0.05 \%$ ). This measurement result was treated as standard assuming that the transducers are reciprocal [28].

$\mathrm{CW}$ sinewave (fourth type of the excitation signal) was assumed to provide the lowest random errors thanks to pure sinusoidal wave and long acquisition record duration. Unfortunately, it turned out that multiple reflections between transducers cause fluctuation of the received signal (up to $10 \mathrm{~dB}$, refer Fig. 14 for received signal level variation vs. frequency in case of $100 \mathrm{k} \Omega$ load).

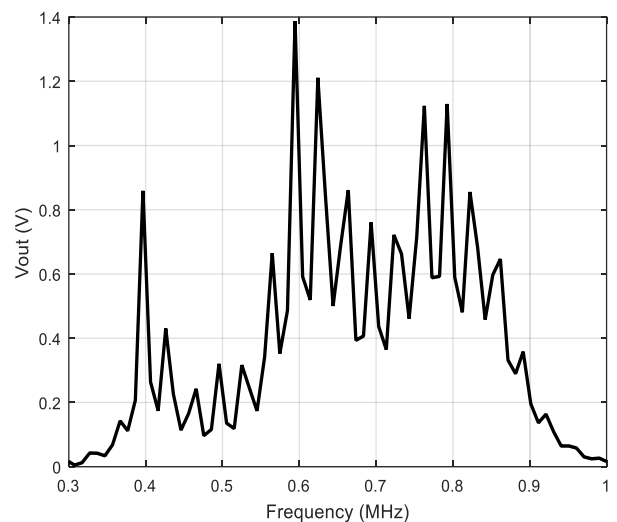

Fig. 14. Received signal level variation over frequency range in case of $\mathrm{CW}$ sinewave excitation due to multiple reflections overlap.

Therefore CW sinewave excitation signal was excluded from further evaluation. Three types used in evaluation are attractive in that sense that no additional equipment has to be added for data acquisition. Refer to Fig. 15 for the example of excitation signal fitted to sinusoid.

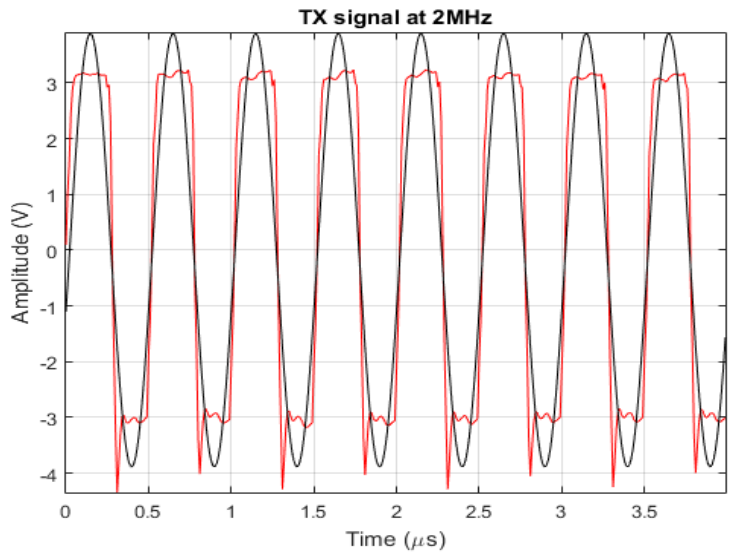

Fig. 15. Rectangular excitation waveform with sinusoid fit.

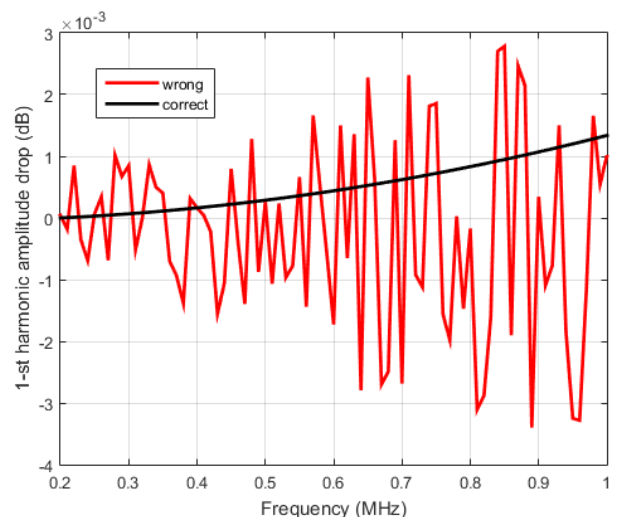

Fig. 16. Variation of fundamental harmonic amplitude in case of correct (black) and wrong (red) frequency grid selection.

It should be noted that rectangular excitation contains certain limitation: amplitude of the fundamental frequency deviates if signal frequency is not an integer fraction of sampling frequency (Fig. 16).

Frequency grid was selected to keep $50 \%$ duty cycle for all frequencies. Transmitting transducer was excited using $\pm 0.3 \mathrm{~V}(0.6 \mathrm{Vpp}), 100 \mu$ s duration rectangular wave in case of chirp and CW tonebursts. In case of pulse excitation, amplitude of the pulse was $0.3 \mathrm{~V}$ and duration was $1.5 \mu \mathrm{s}$, matched to its centre frequency $(650 \mathrm{kHz})$. Same high input impedance amplifier with $40 \mathrm{~dB}$ gain was used for received signal amplification.

\section{A. Transducer Output Impedance Measurement Results}

The third type of excitation signal, rectangular $\mathrm{CW}$ tonebursts of varying fill-in frequency, was assumed to provide best accuracy thanks to high SNR of the received signal. Refer to Fig. 17 for transducer output impedance $\left(R_{L}\right.$ $50 \Omega, R_{H} 330 \Omega$ case) measurement results using this signal.

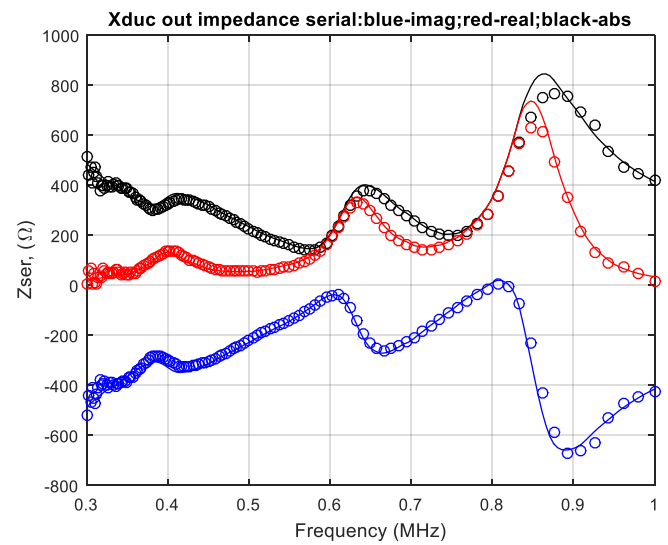

Fig. 17. Transducer output impedance measurement results $\left(R_{L} 50 \Omega, R_{H}\right.$ $330 \Omega$ case) when $\mathrm{CW}$ tonebursts are used for excitation.

Experimental results (circles) are compared with Wayne Kerr 6530B precision impedance analyser results (lines). Refer to Fig. 18 for magnitude errors obtained from Fig. 17.

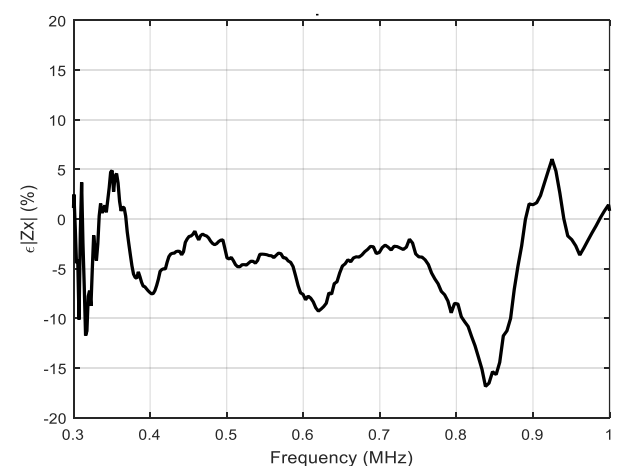

Fig. 18. Transducer output impedance measurement errors when rectangular $\mathrm{CW}$ tonebursts are used for probing, $R_{L} 50 \Omega, R_{H} 330 \Omega$ case.

It can be concluded that error is $10 \%$ at $650 \mathrm{kHz}$ (centre frequency of the transducer). Other combination $\left(R_{L} 330 \Omega\right.$, $R_{H} 10 \mathrm{k} \Omega$ case, Fig. 19, Fig. 20) provide lower errors.

Single probing chirp signal has been used for impedance estimation. In this case, the expected SNR has to be much lower than in case when one $\mathrm{CW}$ toneburst is used per one frequency. Refer to Fig. 21 for single chirp impedance measurement errors (blue line) compared to $\mathrm{CW}$ tonebursts excitation (black). 


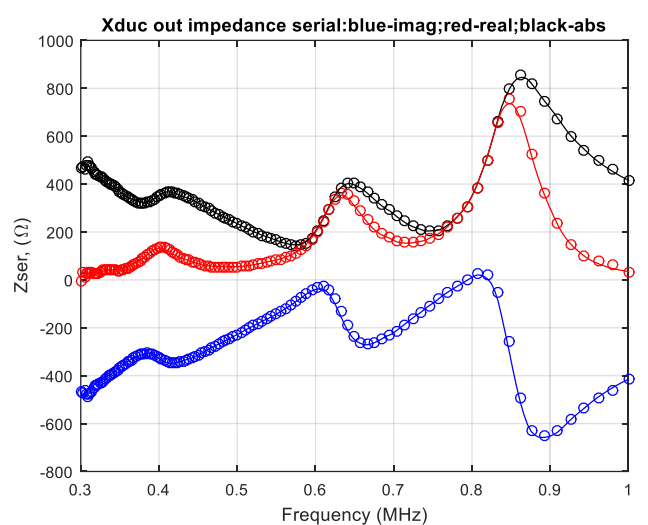

Fig. 19. Transducer output impedance measurement results $\left(R_{L} 330 \Omega, R_{H}\right.$ $100 \mathrm{k} \Omega$ case) when CW tonebursts are used for excitation.

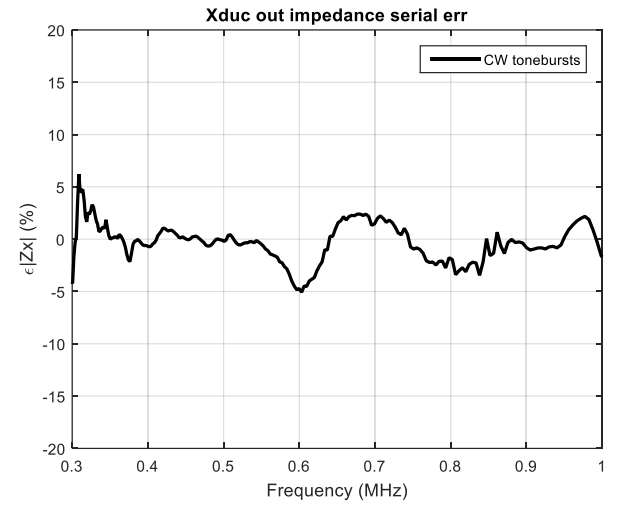

Fig. 20. Transducer output impedance measurement errors when rectangular $\mathrm{CW}$ tonebursts are used, $R_{L} 330 \Omega, R_{H} 100 \mathrm{k} \Omega$ case.

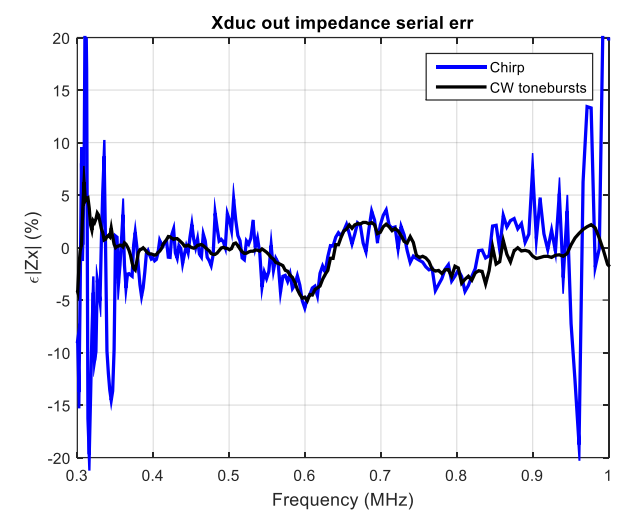

Fig. 21. Transducer output impedance measurement results $\left(R_{L} 330 \Omega, R_{H}\right.$ $100 \mathrm{k} \Omega$ case) when single chirp signal is used for excitation.

It can be seen, that random errors increase in this later case, though, are within $5 \%$ for the main passband, where sufficient transmission is maintained ( $0.4 \mathrm{MHz}$ to $0.9 \mathrm{MHz})$.

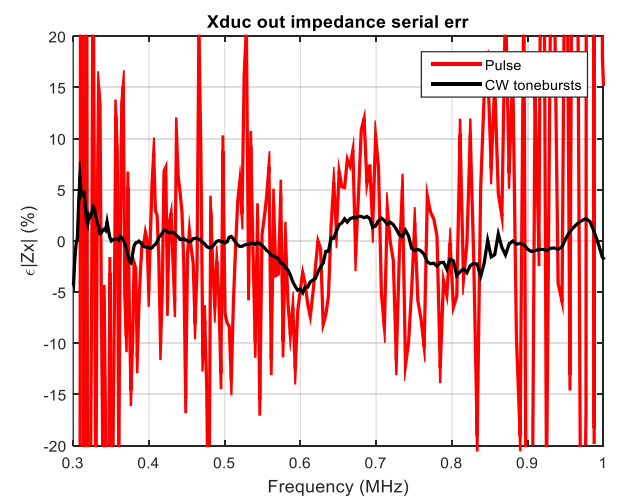

Fig. 22. Transducer output impedance measurement results $\left(R_{L} 330 \Omega, R_{H}\right.$ $100 \mathrm{k} \Omega$ case) when single pulse is used for excitation.
Meanwhile, in the case where single pulse signal was used for excitation, random errors become significant. Refer to Fig. 22 for single pulse (red line) and CW tonebursts (black) errors comparison.

Here SNR is much lower than in case of chirp excitation. Errors obtained are slightly larger than it was predicted from $\left|Z_{x}\right|=300 \Omega$ analysis in chapter II D. With actual impedance data available, detailed error analysis can be carried out.

\section{B. Errors Analysis}

It should be noted, that there was some fluctuation expected in signal transmission over the air. Additional set of experiments has been carried out in order to evaluate how much the results are affected by these air fluctuations. Experiment was repeated 50 times and resulting impedance was evaluated against its average to get the standard deviation of the impedance magnitude (Fig. 23).

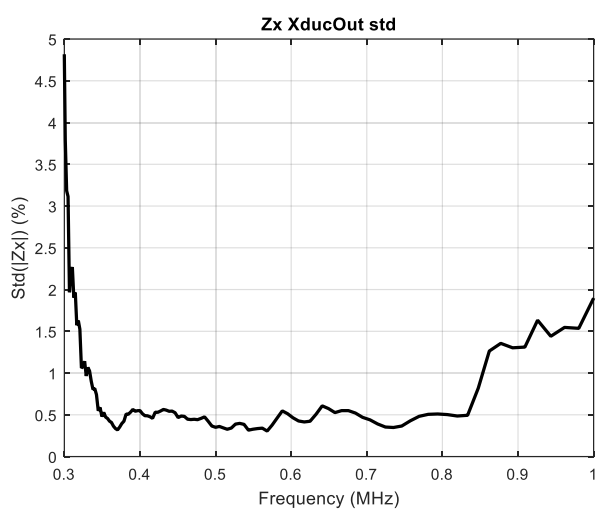

Fig. 23. Transducer output impedance measurement variability with time when $\mathrm{CW}$ tonebursts are used for probing, $R_{L} 50 \Omega, R_{H} 100 \mathrm{k} \Omega$ case.

Measurement also provides intrinsic EMF according to (4). This data, together with output impedance, can be used for errors (Fig. 24) analysis using (1)-(23). Analysis was also used to calculate the optimum $R_{L}$ and $R_{H}$ values. Results for several $R_{L}$ and $R_{H}$ combinations are in Fig. 24.

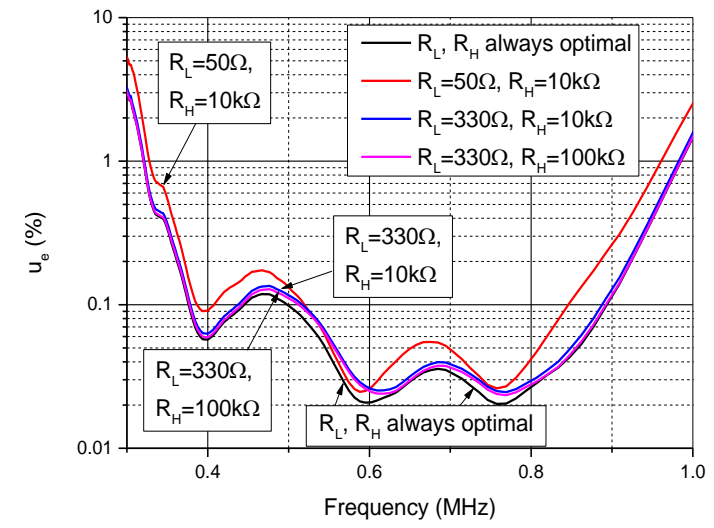

Fig. 24. Expected output impedance measurement uncertainty when $\mathrm{CW}$ tonebursts are used for probing.

It can be seen that only $R_{L} 50 \Omega$ case has slightly larger errors, though all errors do not exceed $1 \%$ within the main passband. Larger deviation obtained in Fig. 18, Fig. 20, Fig. 21 and Fig. 22 can be explained by transducer impedance variation with temperature. Figure 25 is used to demonstrate the impedance variation with temperature. Measurements were carried out using Wayne Kerr 6530B precision impedance analyser, when transducer was kept in 
hand for $5 \mathrm{~min}$ (line err1), then left for cooling for $20 \mathrm{~min}$ (line err2) and for $60 \mathrm{~min}$ (line err3). Reference for errors calculation was the measurement result after transducer was left under room temperature for $2 \mathrm{~h}$.

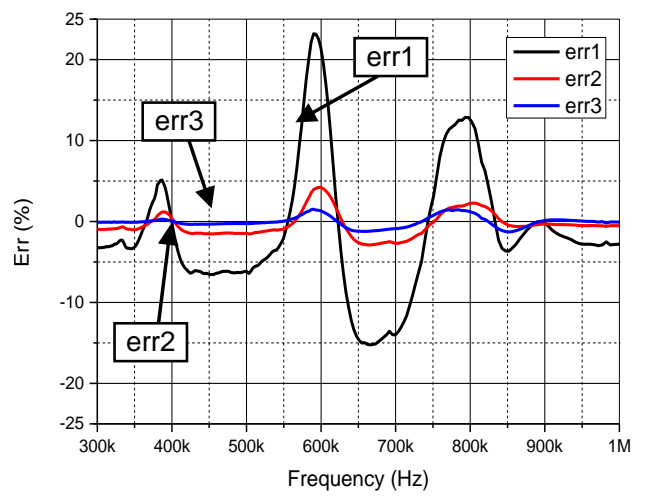

Fig. 25. Output impedance variation with temperature.

It can be concluded that impedance estimation errors are within $3 \%$ expanded uncertainty, though thermal variation of the impedance and air propagation fluctuation do not allow to carry out the measurement with less than $5 \%$ errors.

\section{CONCLUSIONS}

The output impedance measurement technique proposed in this paper is simple, requires only one channel and bias errors in the voltage measurement are low (no calibration). In addition, there is no need for specialized impedance measurement or data acquisition system, as conventional ultrasonic systems and simple excitation signals can be used for measurements. It is ground referenced, so there is no need of removing the transducer from the positioning fixture. High accuracy (expanded uncertainty less than $3 \%$ ) was obtained without calibration. Low voltage measurement random errors ensure wide dynamic range, no need for reference resistor adaptation.

\section{REFERENCES}

[1] I. Amenabar, A. Mendikute, A. Lopez-Arraiza, M. Lizaranzu, J. Aurrekoetxea, "Comparison and analysis of non-destructive testing techniques suitable for delamination inspection in wind turbine blades", Composites B, vol. 42, no. 5, pp. 1298-1305, 2011. DOI: 10.1016/j.compositesb.2011.01.025.

[2] A. Katunin, K. Dragan, M. Dziendzikowski, "Damage identification in aircraft composite structures: A case study using various nondestructive testing techniques", Composite structures, vol. 127, pp. 1-9, 2015. DOI: 10.1016/j.compstruct.2015.02.080.

[3] A. Mizrach, "Ultrasonic technology for quality evaluation of fresh fruit and vegetables in pre- and postharvest processes", Postharvest biology and technology, vol. 48, no. 3, pp. 315-330, 2008. DOI: 10.1016/j.postharvbio.2007.10.018.

[4] T. H. Gan, D. A. Hutchins, D. R. Billson, D. W. Schindel, "The use of broadband acoustic transducers and pulse-compression techniques for air-coupled ultrasonic imaging", Ultrasonics, vol. 39, no. 3, pp. 181-194, 2001. DOI: 10.1016/S0041-624X(00)00059-7.

[5] D. E. Chimenti, "Review of air-coupled ultrasonic materials characterization", Ultrasonics, vol. 54, no. 7, pp. 1804-1816, 2014. DOI: $10.1016 /$ j.ultras.2014.02.006.

[6] M. M. Saad, C. J. Bleakley, T. Ballal, S. Dobson, "High-accuracy reference-free ultrasonic location estimation", IEEE Trans. Instrum. Meas., vol. 61, pp. 1561-1570, 2012. DOI: 10.1109/TIM.2011.2181911.

[7] T. E. G. Alvarez-Arenas, E. Gil-Pelegrin, J. Ealo Cuello, et al.
"Ultrasonic sensing of plant water needs for agriculture", Sensors, vol. 16, no. 7-1089, 2016. DOI: 10.3390/s16071089.

[8] M. G. L. Roes, J. L .Duarte, M. A. M. Hendrix, E. A. Lomonova, "Acoustic energy transfer: a review", IEEE Trans. Ind. Electron., vol. 60, no. 1, pp. 242-248, 2013. DOI: 10.1109/TIE.2012.2202362.

[9] T. E. G. Alvarez-Arenas, "Magnitude and phase spectral analysis of through-transmitted ultrasound pulses for the determination of the ultrasound velocity and the thickness of solid plates", Physics Procedia, vol. 3, pp. 541-550, 2010. DOI: 10.1016/j.phpro.2010.01.070.

[10] T. E. G. Alvarez-Arenas, "Air-coupled piezoelectric transducers with active polypropylene foam matching layers”, Sensors, vol. 13, no. 5, pp. 5996-6013, 2013. DOI: 10.3390/s130505996.

[11] V. Bovtun, J. Doring, J. Bartusch, A. Erhard, Y. Yakymenko, "Ferroelectret non-contact ultrasonic transducers", Appl. Phys. A, vol. 88, pp. 737-743, 2007. DOI: 10.1007/s00339-007-4060-3.

[12] J. Salazar, A. Turo, J. A. Chavez, J. A. Ortega, M. J. Garcia, "Highpower high-resolution pulser for air-coupled ultrasonic NDE applications", IEEE Trans. Instrum. Meas, vol. 52, pp. 1792-1798, 2003. DOI: 10.1109/TIM.2003.820445.

[13] L. Svilainis, A. Chaziachmetovas, V. Dumbrava, "Efficient high voltage pulser for piezoelectric air coupled transducer", Ultrasonics, vol. 53, no. 1, pp. 225-231, 2013. DOI 10.1016/j.ultras.2012.06.004.

[14] J. K. Poulsen, "Low loss wideband protection circuit for high frequency ultrasound", in Proc. IEEE Ultrasonics Symposium, 1999 pp. 823-826. DOI: 10.1109/ULTSYM.1999.849523.

[15] H. Choi, K. K. Shung, "Protection circuits for very high frequency ultrasound systems", J Med Syst, vol. 38, no. 4-34, 2014. DOI: 10.1007/s10916-014-0034-0.

[16] J. Camacho, C. Fritsch, "Protection circuits for ultrasound applications", IEEE Trans. Ultrason., Ferroelect., Freq. Contr., vol. 55, no. 5, pp. 1160-1164, 2008. DOI 10.1109/TUFFC.2008.769.

[17] A. Turo, J. Salazar, J. A. Chavez, H. B. Kichou, T. E. Gomez, F. Montero de Espinoza, M. J. Garcia-Hernandez, "Ultra-low noise front-end electronics for air-coupled ultrasonic non-destructive evalution", NDT\&E International, vol. 36, pp. 93-100, 2003. DOI: 10.1016/S0963-8695(02)00091-9

[18] Y. Yanez, M. J. Garcia-Hernandez, J. Salazar, A. Turo, J. A. Chavez, "Designing amplifiers with very low output noise for high impedance piezoelectric transducers", NDT\&E International, vol. 38, pp. 491496, 2005. DOI: 10.1016/j.ndteint.2005.01.004.

[19] C. D. Motchenbacher, J. A. Connelly, Low-noise electronic system Design. Wiley-Interscience. 1993.

[20] L. Svilainis, V. Dumbrava, D. Kybartas, "Evaluation of the ultrasonic preamplifier noise voltage density", Journal of Circuits, Systems and Computers, vol. 23, no. 1-1450007, 2014. DOI: $10.1142 / \mathrm{S} 0218126614500078$

[21] L. Svilainis, V. Dumbrava, S. Kitov, A. Aleksandrovas, P. Tervydis, D. Liaukonis, "Electronics for ultrasonic imaging system", Elektronika ir Elektrotechnika, vol. 20, no. 7, pp. 51-56, 2014. DOI: 10.5755/j01.eee.20.7.8024.

[22] A. Kalashnikov, V. G. Ivchenko, R. E. Challis, B. R. Hayes-Gill "High-accuracy data acquisition architectures for ultrasonic imaging", IEEE Trans. Ultrason., Ferroelect., Freq. Contr., vol. 54, no. 8, pp. 1596-1605, 2007. DOI: 10.1109/TUFFC.2007.430.

[23] P. K. Weber, H. Fonfara, H. J. Welsch, D. Schmitt, C. Gunther, "A phased array system for the acquisition of ultrasonic RF-data up to 20 MHz", Acoustical Imaging, vol. 27, pp. 25-32, 2004. DOI: 10.1007/978-1-4020-2402-3_4.

[24] S. Cochran, Piezoelectricity and basic configurations for piezoelectric ultrasonic transducers. Woodhead Pub., 2012, ch. 1. DOI: 10.1533/9780857096302.1.3.

[25] Impedance Measurement Handbook. Agilent Technologies. USA. 2003. pp. 1-128.

[26] Eight Hints for Successful Impedance Measurements. App. Note 3464. Agilent Technologies. USA. 2000. pp. 1-28.

[27] L. Svilainis, A. Rodriguez-Martinez, A. Chaziachmetovas, A. Aleksandrovas, "Ultrasound transmission spectral compensation using arbitrary position and width pulse sets", IEEE Trans. instrum. Meas., vol. 67, iss. 8, 2019. DOI: 10.1109/TIM.2018.2809838.

[28] K. K. Andersen, P. Lunde, J. Kocbach, "Magnitude and phase reciprocity calibration of ultrasonic piezoelectric disk in air", in Proc. Scandinavian Symposium on Physical Acoustics, Geilo, Norway, 2016, pp. 1-21. 